\title{
Drogue et transgressions sociales
}

Les femmes et l'opium à Canton dans les années 1930

Drugs and social transgressions: women and opium in Canton in the 1930s

\section{Xavier Paulès}

\section{CpenEdition}

Journals

Édition électronique

URL : https://journals.openedition.org/clio/8732

DOI : $10.4000 /$ clio.8732

ISSN : 1777-5299

Éditeur

Belin

Édition imprimée

Date de publication : 15 décembre 2008

Pagination : 223-242

ISSN : $1252-7017$

Référence électronique

Xavier Paulès, «Drogue et transgressions sociales », Clio. Histoire, femmes et sociétés [En ligne], $28 \mid$

2008, mis en ligne le 15 décembre 2011, consulté le 24 avril 2022. URL : http://

journals.openedition.org/clio/8732 ; DOI : https://doi.org/10.4000/clio.8732 


\title{
Varia
}

\section{Drogue et transgressions sociales Les femmes et l'opium à Canton dans les années 1930*}

\author{
Xavier PAULÈS
}

Les historiens chinois classiques comme Sima Qian réservent une place de choix au thème de la belle femme qui, par son ascendant sur le Souverain, provoque une cascade de désastres politiques ${ }^{1}$. Mais il ne s'agit là que d'un cas de figure découlant du principe plus général selon lequel la passion que peuvent inspirer les femmes est susceptible d'écarter les hommes d'une conduite droite. Les redoutables prédatrices sexuelles dont la littérature propose un choix assez large témoignent aussi du fait qu'elles savent tirer de la sexualité un pouvoir aliénant ${ }^{2}$.

D’une façon comparable, quoique plus récemment (le début de sa diffusion en Chine ne date que du XVIII siècle), l'opium s'est trouvé désigné comme un agent potentiel de déstabilisation du corps social. Selon ses contempteurs, les plaisirs de la drogue détournent de leurs devoirs les plus élémentaires les consommateurs masculins. Ainsi, dans

* L'auteur remercie Stéphane Peltan et Xiao Chunyi pour leur aide respective. Par ailleurs, cet article n'aurait pu voir le jour sans la générosité de la Japan Society for the Promotion of Science (JSPS) et celle de la fondation Chiang Ching-Kuo.

1 Sima Qian 1967. On trouve le thème de la «ravageuse » des hautes sphères politiques dès le VIII ${ }^{e}$ siècle avant J.-C. (Shijing, Classique des poèmes : livre III, chant X, livre IV chants VIII et IX).

2 On pense à Pan Jinlian, personnage-clé du grand roman Fleur en fiole d'or. Le mythe des femmes-renardes (bulijing), créatures redoutables dont les charmes peuvent se révéler destructeurs pour les hommes qui y succombent fait lui aussi écho à l'usage de la sexualité féminine à des fins aliénantes : Pu Songling 1996. 
un pays où la piété filiale constitue l'une des valeurs fondamentales et où la famille fait figure de clé de voûte de l'ordre social, le fumeur aveuglé par sa dépendance est accusé de négliger ses parents et ses proches, allant jusqu'à vendre épouse et enfants pour se procurer sa dose $^{3}$.

Se dessine donc une intéressante convergence entre femmes (ou plutôt leur beauté qui peut susciter un désir incontrôlable) et opium, tous deux capables de maintenir les hommes sous leur emprise, mettant ainsi en péril un ordre social fondé sur la domination masculine. Mais comment ces deux menaces potentielles interagissent-elles ? Étudiés par Keith McMahon, les textes littéraires du XIX ${ }^{e}$ siècle traitant de l'opium distinguent trois possibilités ${ }^{4}$. Selon la première, une menace annule l'autre : l'emprise exercée par l'opium sur ses adeptes serait telle que la compagnie des femmes et les plaisirs du sexe (mais aussi d'autres comme ceux de la nourriture) perdraient tout attrait. Selon la seconde, les femmes consommatrices d'opium s'en trouvent virilisées, et susceptibles de se détourner de leur rôle légitime de mères et d'épouses dévouées, la consommation excessive d'opium pouvant même les rendre incapables d'enfanter. Enfin, le troisième cas de figure évoque l'instrumentalisation de la drogue, où l'opium devient un auxiliaire des visées séductrices de la femme (souvent une prostituée).

Comme l'étude citée, les rares recherches qui ont traité des relations entre les femmes et l'opium en Chine se sont seulement penchées sur les prostituées et les femmes des élites au XIXe siècle, en se basant quasi exclusivement sur des sources littéraires ${ }^{5}$. Le présent article se propose de reprendre la question en élargissant la perspective à l'ensemble de la société. Pour cela, il apparait nécessaire d'abandonner le XIXe siècle, qui n'offre guère de sources sur la consommation dans des milieux populaires, au profit du XXe, et de renoncer au projet d'embrasser l'ensemble du pays. Un net clivage oppose en effet les provinces de l'intérieur qui produisent le pavot (comme le Sichuan, le Guizhou ou le

3 Chinese repository, juin 1838, p. 107-109; Royal Commission on opium 1894 ; Tubua ribao (Le quotidien illustré), $\mathrm{n}^{\circ} 346,355,358,363,365,380,396$ (1910).

McMahon 2002.

5 McMahon 2002 ; Zheng 2005. 
Yunnan), pays de cocagne où l'opium, surabondant et bon marché, est consommé de façon très large, et les provinces comme le Guangdong, où le pavot ne pousse guère et où la drogue ne parvient qu'au terme de longs trajets induisant un surcoût considérable, frein à la consommation ${ }^{6}$. De plus, au sein d'une même province, le contexte des grandes villes est fort différent de celui des campagnes reculées ${ }^{7}$. Pour ces raisons, et pour disposer de sources suffisamment abondantes et variées, il est sage de restreindre l'étude à l'un des centres urbains majeurs du pays.

Le choix de Canton s'avère pertinent pour deux raisons: tout d'abord, il contribue à une meilleure connaissance d'une métropole très peu étudiée, surtout par rapport à Shanghai. D'autre part, Canton compte dans les années 1930 un journal qui accorde une importance insolite à la consommation d'opium dans les éditoriaux, les contributions de lecteurs et surtout les faits divers: le Yuehuabao. Les faits divers ne diffèrent pas fondamentalement de ce que nous connaissons aujourd'hui en Occident. Il s'agit de brefs comptes rendus de petits événements qui viennent rompre la monotonie du quotidien. Ceux qui se rapportent à l'opium, licite à cette époque, relatent typiquement les circonstances de vols, escroqueries ou bagarres survenues dans les fumeries ou encore l'arrestation de fumeurs d'opium de contrebande. Outre le Yuehuabao, il est possible d'utiliser des articles publiés dans les revues cantonaises généralistes et les revues anti-opium (Judu yuekan, Jinyan banyuekan), ainsi que des entretiens avec des témoins de l'époque.

\section{Fumeuses d'opium : représentations et réalités}

Selon les œuvres littéraires de la fin des Qing (1850-1911), pour les raisons que nous avons signalées, les fumeuses sont condamnées plus sévèrement que leurs homologues masculins. En dehors du champ littéraire, dans des publications hostiles à l'opium, on trouve aussi, même s'il ne s'agit jamais d'une thématique centrale, l'idée que la

6 China weekly review, 23 septembre 1933, p. 148 ; Royal Commission on opium 1894.

7 Rapport du docteur Hobson à Sir J. Bowring du 6 novembre 1855: British Parlementary Papers 1971. 
consommation par les femmes représente un stade extrême des ravages de la drogue ${ }^{8}$. Ces sources traduisent une certaine inquiétude du milieu où elles sont produites, à savoir l'élément mâle des élites, devant la consommation des femmes de leur strate sociale.

La lecture de la presse et des périodiques cantonais des années 1930 laisse une impression quelque peu différente. Les faits divers concernant les fumeuses, très fréquents, s'indignent volontiers de l'inconduite des femmes concernées. Mais les nombreuses diatribes anti-opium, elles, ne ménagent qu'une place négligeable au thème des ravages de l'opium parmi les femmes. Le sujet est pratiquement passé sous silence dans les nombreux écrits de propagande publiés à Canton en 1936-1937 par le Guomindang9. Cette littérature désigne toujours le fumeur sans détermination de genre, usant de termes comme xishi de ren (les fumeurs), ou xiyan de ren (les fumeurs d'opium). Mais en réalité, il s'agit toujours implicitement d'un homme, comme on ne tarde pas à s'en apercevoir lorsque survient par exemple une référence à la vente de son épouse pour satisfaire ses besoins en opium. Un texte de la même époque qui expose les différents moyens à employer pour parvenir a une éradication rapide de l'opium invite les enfants à inciter leur père (et les femmes leur mari) à s'abstenir de fumer ${ }^{10}$. Lorsqu'une trajectoire typique de fumeur est mise en scène, il s'agit toujours d'un homme, plutôt jeune et fortuné, dont l'épouse n'apparait que pour illustrer les déboires de la famille (voir Cahier d'images, figure 10). La femme est donc présentée presque exclusivement comme une victime collatérale de la consommation d'opium.

Si l'abondance des faits divers mettant en scène des fumeuses et l'absence du thème des ravages de l'opium parmi les femmes dans la

8 Tubua ribao, $\mathrm{n}^{\circ} 374$ (1910), p. 5 ; Shishi buabao (L'actualité illustrée), septembre 1907, p. $10 \mathrm{~b}$.

9 À cette époque, une intense activité de propagande est orchestrée au niveau national par le Guomindang dans le cadre d'une campagne visant à éradiquer l'opium dans un délai de six ans. Un grand nombre de tracts, discours et écrits produits à Canton par l'organisme ad hoc se trouvent rassemblés dans une imposante compilation : Guangzhoushi jinyan weiyuanhui 1937.

10 Guomindang guangdongsheng dangbu, Jinyandu z̧huankan (Édition spéciale pour la suppression de l'opium et du jeu), p. 37. 
propagande paraissent à première vue contradictoires, ils découlent pourtant du même phénomène: la réprobation spécifique de la consommation féminine. La consommation illégale par des femmes est jugée digne de mention précisément parce qu'elle revêt un aspect sensationnel. Du reste, les femmes arrêtées pour des délits en rapport avec l'opium manifestent presque systématiquement la honte qu'elles ressentent face à une foule hostile lors de leur transfert au poste de police, dissimulant leur visage, un état de fait rarissime quand des hommes sont appréhendés pour les mêmes raisons ${ }^{11}$.

Quasi universelle, la stigmatisation des fumeuses explique aussi la très faible importance de la consommation par les femmes et que, par conséquent, la propagande cible de préférence la consommation masculine. Leur cas est tout à fait comparable à celui des enfants, notoirement absents, eux aussi, de la propagande ${ }^{12}$. Dans ces deux cas, il apparait inutile de mobiliser les moyens limités de la propagande pour une bataille en réalité déjà gagnée. Passer sous silence le cas des femmes (aussi bien que celui des enfants) est un choix pragmatique qui permet de cibler ceux qui forment les gros bataillons des fumeurs : les hommes adultes.

Une étude systématique des fumeurs à Canton dans les années 1930 a permis de conclure qu'environ un fumeur cantonais sur dix est une femme. Sachant que le pourcentage de fumeurs parmi la population totale tourne vraisemblablement autour de $3 \%$ à $4 \%$, on peut se figurer à quel point la consommation féminine représente un phénomène marginal ${ }^{13}$. Lors des entretiens, les témoins de l'époque confirment, voire incitent à forcer le trait en affirmant de façon unanime que les

11 Yuehuabao (Le méridional, ci-après : YHB) 10/5/30, 18/10/30, 5/6/31, 11/5/31, $6 / 3 / 32$.

12 Dans le cas des enfants, la consommation d'opium est rarissime en raison de croyances solidement enracinées selon lesquelles celui qui commence à fumer avant d'avoir achevé sa croissance mourra prématurément.

13 Paulès 2005a. 
femmes ne fumaient pas. Aucun ne connait de cas de fumeuse d'opium dans son entourage ${ }^{14}$.

La façon très négative dont est perçue la consommation féminine n'explique pas seule qu'un nombre très faible de femmes fument. Deux autres raisons peuvent être évoquées. Premièrement, des facteurs économiques s'opposent à la consommation d'opium parmi les femmes des couches populaires. Des enquêtes sociologiques précises indiquent que la dépense que représente la consommation régulière d'opium ou même de dérivés moins coûteux dans le budget d'une famille modeste est loin d'être négligeable. Prenons l'exemple d'une étude réalisée parmi la population des Tankas ${ }^{15}$ de la zone de Shanan, particulièrement précieuse parce qu'elle fournit des données chiffrées à la fois sur la consommation d'opium et sur les revenus ${ }^{16}$. Même les besoins d'un fumeur modéré, de l'ordre de 0,2 yuan par jour, représentent pas moins de $13 \%$ du revenu moyen d'une famille modeste. Par conséquent, si dans certaines familles de coolies le chef de famille a recours à l'opium pour pouvoir mener à bien le travail épuisant qui représente toujours la principale ressource du foyer ${ }^{17}$, leurs femmes mettraient gravement en péril la survie du ménage si elles se laissaient aller, elles aussi, à fumer. Intériorisée, cette contrainte économique a pu du reste renforcer dans les milieux populaires l'idée qu'il n'est pas convenable qu'une femme fume ${ }^{18}$.

14 Entretiens avec sept témoins menés les 5 octobre 2005, 7, 10 et 11 juillet 2006, 15 juillet 2008. Étant donné la réprobation spécifique dont sont l'objet les fumeuses, on ne peut néanmoins pas écarter le risque d'auto-censure.

15 Les Tankas sont alors une population de réprouvés, à l'origine controversée, qui vit de façon permanente dans des embarcations sur la rivière des Perles.

16 Lingnan Shehui Yanjiusuo 1934.

17 L'opium est consommé très fréquemment dans des métiers particulièrement pénibles comme celui de tireur de pousse-pousse, car il permet de lutter contre la sensation de fatigue: Murayama 1941 ; Judu yuekan (Mensuel pour la suppression des drogues), $n^{\circ} 90$ (juin 1935), p. 7 ; YHB 5/5/33.

18 Ce mécanisme d'intériorisation d'une contrainte objective, "nécessité faite vertu ", renvoie bien évidemment au concept d'habitus élaboré par Bourdieu (Bourdieu 1979). 
La seconde raison découle du changement d'attitude des élites vis-àvis de l'opium. En effet, des témoignages assez nombreux faisaient état, à la fin de la période impériale, du fait que les fumeuses étaient principalement des femmes de riches familles (et des prostituées) ${ }^{19}$. Or, on assiste à Canton, entre la fin du XIX siècle et les années 1930, à une mutation de la population des fumeurs d'opium. Quantitativement, le pourcentage de fumeurs parmi la population totale subit une baisse considérable, passant de 10 à environ $4 \%$. Par ailleurs, qualitativement, les catégories sociales les plus favorisées, particulièrement réceptives au discours qui lie le déclin de la Chine à l'influence de l'opium, se détournent de la drogue ${ }^{20}$. Il en résulte une paupérisation des rangs de fumeurs parmi lesquels, dans les années 1930, coolies et ouvriers sont largement sur-représentés. Du fait que les catégories de la population qui se détournent de la drogue sont celles qui comptaient auparavant, en proportion, le plus de fumeuses, la consommation féminine s'en trouve par conséquent plus spécifiquement diminuée.

Un dernier indice suggère que, dans les représentations collectives, la femme fumeuse a cessé sous la République de représenter une menace crédible. On constate en effet qu'en matière de rapport entre femmes et opium, l'attention de la société se porte largement moins sur les consommatrices que sur la question des yanhua («fleurs de la fumée »).

\section{La question de l'interdiction des yanhua}

La préparation d'une pipe d'opium requiert à la fois du doigté et du temps. De plus, l'opération doit être répétée pour autant de pipes que nécessaire, étant entendu qu'un consommateur moyen en fume une petite dizaine par jour ${ }^{21}$. La fonction des yanbua consiste précisément à épargner aux clients des fumeries cette corvée, en présentant les pipes toutes prêtes, et à leur tenir compagnie en leur faisant la conversation. Elles peuvent aussi leur proposer des cigarettes et des douceurs.

\footnotetext{
Royal Commission on opium 1894.

Paulès 2005b.

Paulès 2005b.
} 
Probablement parce que ces jeunes et jolies femmes intéressent son lectorat, essentiellement masculin, différents types d'articles du Yuebuabao, notre principale source, font référence aux yanbua. Certains racontent une visite dans une fumerie durant laquelle l'attention de l'auteur a été attirée par une yanbua qui y officie. Il se renseigne auprès du personnel, d'une connaissance ou d'un voisin qui généralement ne se font guère prier pour raconter l'histoire de la jeune femme. De tels articles ont peut-être tendance à romancer, c'est pourquoi les faits divers concernant des vols ou des bagarres dans les fumeries qui mentionnent en passant une yanbua quand elle s'y trouve impliquée d'une manière ou d'une autre sont, en dépit de leur brièveté, tout à fait précieux : ils livrent une information probablement plus objective sur leur quotidien ${ }^{22}$. Le troisième type d'articles sont des réquisitoires contre les yanbua. La question de leur interdiction est ainsi un thème récurrent dans le Canton des années 1930, qui s’inscrit d'ailleurs dans le problème plus large des nü zhaodai, terme qui désigne les employées chargées du service dans fumeries, restaurants et maisons de thé23.

En 1930-31, les autorités proclament à diverses reprises l'interdiction des yanbua ${ }^{24}$. Néanmoins, elles réapparaissent toujours dans les fumeries, quitte à contourner la loi en se faisant par exemple passer pour des vendeuses de fruits ${ }^{25}$. Durant les deux premiers mois de l'année 1932, on se passionne pour la question de l'interdiction des yanhua qui sont la cible d'une véritable campagne de presse dans le Yuebuabao ${ }^{26}$. Les articles accusateurs se multiplient. Ils insistent sur la

22 Voir par exemple YHB 15/2/31, 12/1/32, 9/3/35, 17/11/36. Parfois aussi, de tels fait divers deviennent l'occasion d'évoquer brièvement la trajectoire de la yanbua en question: YHB 29/2/34, 24/11/34, 28/7/35

23 Chin 2007.

24 YHB 24/3/30, 30/5/30, 18/11/31, 21/11/31; Guangzhoushi shiæheng gongbao (Le journal officiel du gouvernement de la municipalité de Canton), novembre 1930.

25 Yugong sanrikan (Le journal de Yugong), $\mathrm{n}^{\circ} 31, \mathrm{n}^{\circ} 60$ (ca 1930); YHB 11/12/31, $10 / 1 / 32,4 / 2 / 32$.

26 Pourtant, il faut remarquer que le journal Guangzhou minguo ribao (Le quotidien démocratique de Canton), très proche des autorités, ne se fait pas alors l'écho de tels débats et que, de façon difficilement explicable, il ne mentionne même pas la mesure prise d'interdire les femmes dans les fumeries. 
nécessité impérative de mettre terme une fois pour toutes à la présence de ces femmes dans les fumeries ${ }^{27}$. Voici, représentative de cette prose, la conclusion d'un article de janvier 1932 :

En définitive, les yanhua portent grandement atteinte aux mœurs et causent bien des problèmes. Cela est plus particulièrement vrai pour la jeunesse au sang chaud et les étudiants qui, désertant les cours, viennent traîner dans les fumeries pour convoiter les yanhua. Beaucoup deviennent des drogués de cette façon. C'est une chose très regrettable et il faut espérer une véritable et stricte interdiction [des yanbua], sans qu'elles puissent renaittre sous aucune autre forme. Cela serait là un bienfait inappréciable pour la jeunesse! 28

Comme dans le cas présent, les attaques reposent principalement sur deux arguments : les yanbua ont, d'une façon générale, une attitude indécente et d'autre part elles tendent à détourner les hommes du travail productif pour les attirer vers la drogue. L'inquiétude est d'autant plus vive que les «victimes» des yanbua sont jeunes. Nous reviendrons sur les accusations d'«indécence». Quant au nombre de non-fumeurs venus à la drogue à cause des yanhua, il est impossible à évaluer. Les yanbua constituant un argument commercial utilisé par les patrons pour augmenter l'affluence ${ }^{29}$, leur impact ne doit pas être négligeable, surtout si l'on estime que chacune des 300 fumeries cantonaises possède une ou plusieurs yanbua ${ }^{30}$.

Quoiqu'il en soit, la campagne de presse hostile aux yanbua atteint son but puisqu'au mois de mars 1932, une décision radicale est prise : l'interdiction totale des femmes, clientes et employées, dans les fumeries ${ }^{31}$. La mesure porte aussitôt un coup assez rude aux affaires de ces établissements ${ }^{32}$. Dans les années qui suivent, l'interdiction

YHB 7/1/32, 10/1/32, 12/1/32, 15/1/32, 2/2/32, 4/2/32, 11/2/32.

YHB 15/1/32.

Yugong sanrikan, $\mathrm{n}^{\circ} 53$ (ca 1930); Huari ribao 24/8/29; YHB 5/12/31, 10/1/32.

30 YHB 11/12/31. A propos de l'estimation de 300 fumeries pour la ville de Canton : Paulès, 2005b. Le seul ordre de grandeur présent dans les sources concernant le nombre de yanbua de la ville s'élève à « plusieurs centaines »: YHB 22/6/32.

31 YHB 22/3/32.

32 Huari ribao (Le journal Huazi) 4/6/32, YHB 22/3/32, 22/6/32, 9/6/34. 
continue à être appliquée ${ }^{33}$. Des indices de relâchement sont cependant détectables vers 1934-35, mais il ne s'agit pas, semble-t-il, d'un retour massif et au grand jour des yanbua. Les nouvelles des journaux et des revues apparaissent contradictoires : dans la même période, elles font état d'une part d'arrestations de femmes surprises et interpellées dans des fumeries ${ }^{34}$, et d'autre part de la présence tout à fait ouverte de clientes et de yanhua dans certains établissements chics ${ }^{35}$. Cette situation quelque peu confuse se perpétue jusqu'à la fin de la domination de l'autocrate Chen Jitang ${ }^{36}$ à l'été 1936. Dans les mois qui suivent sa chute, les autorités du Guomindang de retour au Guangdong réaffirment sans ambiguïté l'interdiction stricte des yanbua ${ }^{37}$.

\section{Les yanhua: prostituées ou « marchandes de face " ?}

Si la raison d'être des yanbua est de préparer l'opium à la place des clients et de leur tenir compagnie, de nombreux articles de presse font allusion à leur attitude indécente. Certains assimilent les yanbua à des prostituées clandestines, un rapprochement que le terme de «fleur de la fumée » invite à faire ${ }^{38}$. Il faut encore ajouter que la consommation d'opium, en raison d'une utilisation ancienne dans les bordels pour calmer le désir et prolonger la durée des rapports sexuels, mais aussi de son insertion subtile dans les relations sociales de ces établissements, se

33 Elle s'accompagne aussi du renvoi de certains commissaires de police coupables d'un laxisme excessif sur ce chapitre, ce qui a certainement stimulé le zèle de leurs collègues restés en place : YHB 22/3/32.

34 YHB 17/1/34, 9/3/34, 22/11/34, 24/11/35.

35 Deux articles du Judu yuekan, $\mathrm{n}^{\circ} 79$ (circa 1934), p. 31, et $\mathrm{n}^{\circ} 90$ (juin 1935), p. 5-7 mentionnent la présence de nombreuses femmes comme clientes et comme yanhua dans les fumeries de luxe de Canton.

36 Chen Jitang (1890-1954) est un chef militaire du Guomindang qui parvient, à partir de 1931, à s'assurer le contrôle de la province du Guangdong. Il l'administre de façon indépendante jusqu'à ce que le gouvernement central de Nankin parvienne à l'évincer en 1936.

37 Article $n^{\circ} 30$, règlement du 18 novembre 1936 relatif au fonctionnement de la section des inspecteurs en civil du Comité d'interdiction de l'opium de la ville de Canton, Guangzhoushi jinyan weizuanbui gongzuo jiyao, p. 67.

38 YHB $11 / 12 / 31,27 / 7 / 34$. 
trouve traditionnellement associée dans les représentations collectives à la fréquentation des prostituées ${ }^{39}$. Quelques récits de trajectoires de yanbua mentionnent une activité de prostituée avant ou après qu'elles exercent le métier de yanbua $a^{40}$ et certains phénomènes suggèrent une porosité entre les deux états ${ }^{41}$. Ainsi, après l'interdiction de mars 1932, un certain nombre des jeunes femmes réduites au chômage vont grossir les rangs des prostituées clandestines ${ }^{42}$.

Écrite en 1924, une nouvelle de l'écrivain cantonais Zhang Ziping plaçant résolument le sexe au cœur des rapports entre une yanbua appelée Aju et un de ses clients habituels (Chen Zhongzhang, un petit fonctionnaire véreux) incite effectivement à les rapprocher de ceux d'une prostituée avec son client ${ }^{43}$. Aju espère séduire Chen au point de se faire épouser sans négliger, en attendant, de lui ponctionner le maximum d'argent : outre les pourboires qu'il lui verse, elle prélève sans vergogne une forte commission sur les repas et boissons qu'il commande pour les partager avec elle. Chen, de son côté, n'a nulle intention de la prendre pour femme et s'évertue seulement à coucher avec elle moyennant le moins d'argent possible. Zhang Ziping insiste sur les facilités qu'offre dans les jeux de séduction la situation de deux personnes couchées face à face pour fumer.

Mais, pour une poignée d'articles des années 1930 évoquant sans ambiguité des relations sexuelles entre une yanbua et un client ${ }^{44}$, combien d'articles accusatoires se retranchent derrière des expressions vagues comme «canggounawu» (servir de paravent au vice) 45 ? Certes, une certaine autocensure des journalistes au nom de la bienséance n'est pas à exclure, mais face à des descriptions faisant allusion de façon plus ou moins claire à des relations galantes, ou aux mœurs légères des

Henriot 1997 ; Luo 1994.

YHB 5/8/31, 26/10/31, 20/12/31, 2/2/32, 9/2/32, 5/3/34.

41 Angelina Chin fait le même constat avec les hôtesses des maisons de thé cantonaises : Chin 2007.

42 YHB 11/6/32, 22/6/32, 17/11/32.

43 Zhang Ziping 1994.

YHB 7/9/33, 27/7/34.

5 YHB 30/5/30, 12/1/32, 4/2/32, 17/11/32. 
yanbua, l'historien doit s'abstenir d'y voir systématiquement de la prostitution déguisée ${ }^{46}$.

Il faut replacer les accusations d'« indécence » dans le contexte du Canton de la première moitié des années 1930, qui voit, sous l'impulsion de Chen Jitang, la promotion de valeurs traditionnelles qui avaient été largement battues en brèche dans les années 1910-1920 par des autorités alors presque toujours résolument progressistes. La remise à l'honneur des Classiques dans les écoles primaires en constitue l'un des aspects les plus fameux ${ }^{47}$, comme les mesures prises en faveur de la séparation marquée des hommes et des femmes dans les lieux publics. Si la ségrégation sexuelle (y compris au domicile) était indiscutablement partie constituante d'un ordre social idéalement dérivé des valeurs confucéennes (Wang Di l'a bien montré dans son étude de la vie des rues de Chengdu), les impératifs théoriques s'effaçaient d'eux-mêmes devant les nécessités de la vie quotidienne des familles modestes ${ }^{48}$. La situation à Canton était sans aucun doute la même et, dans les milieux populaires, femmes et hommes se côtoyaient chaque jour. S'il ne faut pas caricaturer les initiatives de Chen Jitang, qui faisaient à l'époque la joie des contributeurs de la revue satirique progressiste Luny $u^{49}$, il n'en reste pas moins que, par exemple, femmes et hommes se voient interdire de se baigner ensemble dans les piscines de plein air ${ }^{50}$ et que la police se montre vigilante pour que les vêtements des femmes se conforment à des règles de décence définies assez strictement ${ }^{51}$. Dans un climat si peu permissif, les yanbua font scandale du simple fait qu'elles côtoient des hommes de près.

Il faut aussi s'entendre sur la notion de prostitution. En effet, ses acceptions chinoise et occidentale ne se recouvrent que très imparfaitement: les études consacrées à ce phénomène en Chine

46 Zhu, Jiang \& Zhang, 1995 assimile ainsi purement et simplement les yanbua cantonaises à des prostituées.

47 Yang \& Zhong, 1996 ; Revue nationale chinoise, mai 1934, p. 398.

48 Wang Di 2003.

49 Lunyu (Propos), n6 (août 1934), p. 1016 ; nº 47 (août 1934), p. 1055 et 1069-71.

Lee 1936.

1 Revue nationale chinoise, octobre 1935, p. 242. 
montrent assez clairement que les rapports sexuels proprement dits ne sont pas, au moins pour l'élite de ce que l'on nomme en français, faute de mieux, les prostituées, au centre des rapports avec le client. S'il serait absurde de nier que certaines yanbua font commerce de leur corps, cela ne signifie nullement que telle soit la raison d'être de la majorité d'entre elles et qu'on doive faire découler leur indiscutable succès de leur seule activité de prostitution. Il semble au contraire que, dans leur grande majorité, les yanbua ne font pas commerce de leur corps ${ }^{52}$, et quand c'est le cas, pas au tout venant. Certaines yanbua choisissent les clients à qui elles donnent rendez-vous dans des hôtels voisins, n’hésitant pas à rabrouer même de riches et généreux barbons ${ }^{53}$. Notons aussi que des patrons de fumeries utilisent leurs propres épouses comme yanbua dans leurs établissements ${ }^{54}$, un argument qui n'est pas définitif, car à l'époque des femmes pauvres se prostituent assez fréquemment avec le consentement de leurs maris ${ }^{55}$.

Pour comprendre le succès des yanhua sans se contenter de voir en elles des prostituées clandestines, il peut être plus éclairant de reformuler le problème en cherchant à savoir pourquoi un consommateur souhaite payer pour être servi par une yanbua. Il est évident tout d'abord que le client qui entre dans une fumerie, est immédiatement servi par une jeune et séduisante yanhua qui effectue à sa place, couchée face à lui, la fastidieuse préparation des pipes d'opium, gagne en agrément ce qu'il va dépenser en pourboires. Le plaisir est ainsi l'explication la plus évidente, mais un article du Yuebuabao du 15 janvier 1932 met en lumière un autre aspect essentiel de la fonction des yanbua qui est d'affirmer, par leur service, le rang du client. Une yanbua du nom de Youbao est en train de s'occuper d'un de ses riches clients habituels, Liu, dans un salon particulier d'une fumerie chic lorsque survient Chen, un autre habitué de ses services, accompagné ce jour là par un ami dont c'est la première visite à cette

52 Un témoin de l'époque, familier des fumeries cantonaises, est affirmatif sur ce point : entretien avec M. Mai Zhaoshen du 15/7/08.

53 YHB 7/1/32.

54 Huazi ribao 24/8/29; YHB 16/11/31, 3/12/31, 20/12/31, 2/8/34.

55 Huaguobao (Le journal du pays) 29/5/15; Henriot 1997. 
fumerie. Si, d'habitude, Youbao se précipite pour lui souhaiter la bienvenue, lui présenter une pipe, épousseter sa couche et lui préparer ses boulettes, cette fois-ci, Chen est désagréablement surpris de ne pas être accueilli par elle. Pire, il fait le pied de grue avec son compagnon dans la salle du rez-de-chaussée car les autres yanbua n'osent pas s'occuper d'un homme qui est une chasse gardée notoire de leur collègue. Indigné d'être ainsi délaissé quand les clients sont entourés des soins des autres yanbua, Chen commence à s'énerver. Il espérait bien montrer à son ami qu'il était traité là comme un client important. Il crie donc pour appeler Youbao que Liu, qui n'accepte pas d'être délaissé au profit d'un rival, refuse de laisser aller. En définitive, les deux hommes en viennent aux mains.

Très parlante, cette anecdote suggère que la yanbua est présente avant tout pour manifester clairement le rang du client. Chen arrive en situation de demande de face ${ }^{56}$, dont il a un besoin d'autant plus impératif qu'il est accompagné. Chacun des deux hommes estime en définitive payer suffisamment pour bénéficier d'un statut de client privilégié par rapport à tous les autres. Leur face-à-face (l'expression française est ici particulièrement adaptée) entraine inévitablement un affrontement. Les sentiments amoureux éventuels ne font que se surajouter aux actes dictés par des considérations de face et des stratégies de prestige. Les élans de la passion, pas plus que ceux du désir sexuel, ne doivent donc occulter le fait qu'une des raisons d'être des yanbua, peut-être même la principale, est en réalité le commerce de face.

Il faut à cet égard revenir sur la distinction de leurs attributions par rapport à celles du reste du personnel des fumeries : n'est-il pas en effet intéressant de remarquer que les yanhua sont les spécialistes de l'accessoire ? Les services qu'elles rendent, à savoir la préparation des pipes (que les consommateurs peuvent effectuer eux-mêmes), l'entretien des clients, mais aussi la vente de friandises ou de cigarettes, viennent manifestement se rajouter à la simple prestation de base. La recherche et le faste avec lesquels elles sont vêtues manifestent

56 La face (mian) désigne en Chine le prestige, la «surface » dont, à tous les niveaux de la société, chacun dispose par rapport à un large entourage. L'expression " perdre la face » que l'on connaît en français est empruntée à la langue chinoise. 
pareillement le rang de celui qui se trouve disposé à s'offrir leurs services ${ }^{57}$. Ce sont, a contrario, les hommes à tout faire (qintong) de la fumerie qui se chargent de distribuer et collecter les pipes, nettoyer les fourneaux, attribuer les couches, encaisser, distribuer le thé, autant de besognes relevant d'un incompressible service minimum. En conséquence, le client se place, dès le moment où il fait appel à une yanhua, dans la position de demandeur d'un service qui excède ce strict minimum. Il s'élève de ce fait au dessus du statut de client de base.

La comparaison du cas des yanbua avec celui des hôtesses des dancings de Shanghai (de jeunes femmes louées par le client pour une danse) s'impose : l'activité des hôtesses est aussi montrée du doigt dans la mesure où elles sont amenées à avoir des contacts rapprochés avec des hommes. Le raccourci est souvent pris qui désigne les dancings comme de simples lieux d'une prostitution déguisée. Mais la thèse d'Andrew Field montre de façon convaincante que pendant les années 1930, très peu d'hôtesses se livrent à la prostitution, car elles peuvent fort bien gagner leur vie simplement en dansant. Cela n'exclut pas qu'une hôtesse choisisse parfois de se livrer à un homme de son choix, libre en cela de mener sa vie sexuelle à son gré. Les hôtesses qui couchent régulièrement avec des clients sont souvent des prostituées qui ont investi les dancings afin d'y retrouver les clients ayant tendance à déserter les bordels, aux règles trop contraignantes ${ }^{58}$.

Comme les danseuses, la plupart des yanbua de fumeries moyennes (et a fortiori de luxe) peuvent gagner correctement leur vie sans avoir recours à la prostitution ${ }^{59}$. Certaines yanhua célèbres, et en tant que telles tout à la fois un enjeu et un arbitre dans les véritables joutes de prestige que se livrent des riches Cantonais pour s'assurer leurs services, peuvent même recevoir sous forme de présents des sommes importantes en peu de temps. Mais sans être des prostituées, toutes ces femmes sont couchées aux côtés des hommes, dans une vraie promiscuité. Là est le vrai objet du scandale, comme les corps enlacés de l'hôtesse de dancing et de son client. Sur les pistes de danse ou dans

YHB 18/11/31, 11/12/31.

Field 2000 ; Henriot 1997.

Paulès 2005b. 
les fumeries, c'est en ce rapprochement banalisé des deux corps de personnes de sexe opposé que réside le nœud du problème.

\section{Trajectoires de yanhua:}

\section{la tentation de la liberté et de l'ascension sociale}

Les articles qui retracent la vie d'une yanbua sont assez nombreux pour que l'on puisse se risquer à caractériser des trajectoires typiques. Les yanbua en activité sont généralement très jeunes, entre 15 et 22 ans le plus souvent, et célibataires. L'origine sociale des jeunes femmes qui se trouvent amenées à exercer ce métier est souvent mentionnée dans les articles qui les concernent. Certains attribuent ainsi le choix délibéré de jeunes femmes de familles aisées en faveur de la profession de yanbua à une «interprétation erronée du concept de liberté (mujie ziyou)» qui leur fait revendiquer, en particulier, une vie sentimentale soustraite au contrôle de leur famille ${ }^{60}$. Néanmoins, même si l'inconduite (à leurs yeux) de ces quelques filles de bonne famille fait les délices des chroniqueurs, il s'avère que le milieu d'extraction des yanbua est généralement modeste ${ }^{61}$. Et des articles assez nombreux mentionnent des filles poussées par la nécessité matérielle à se lancer dans le métier de yanbua ${ }^{62}$.

Il ne semble pourtant pas pertinent d'opposer radicalement des yanbua issues de familles riches qui choisiraient ce métier pour braver les normes sociales pesant sur elles et d'un autre côté de pauvres filles poussées par le besoin. Des trajectoires de yanhua d'origine modeste montrent en réalité souvent la même rupture avec certaines valeurs communément admises par leur milieu d'origine et en particulier une conception traditionnelle de la femme. Associé à une mise élégante, d'un rapport intéressant ${ }^{63}$, le métier de yanhua a aussi vraisemblablement, aux yeux de ces filles, l'attrait supplémentaire d'être,

60 YHB $11 / 2 / 32,5 / 3 / 34$

61 YHB 10/1/32, 31/12/31, 9/2/32, 6/1/34. Selon Mme X, les yanbua travaillant dans la fumerie où elle-même était employée étaient toutes de famille pauvre: entretien avec Mme X du 11/7/06.

62 YHB 10/1/32, 1/5/32, 9/2/32.

63 Plusieurs articles soulignent ce fait: YHB 26/6/31, 26/10/31, 15/1/32. 
en dépit de journées de travail assez longues, dénué de toute pénibilité physique. Lorsque des articles décrivent avec indignation les penchants jugés libidineux de certaines de ces jeunes femmes d'extraction modeste, n'est-il pas plus crédible de mettre en avant leur aspiration à l'autonomie financière, à une vie plus libre et plus confortable que celle d'épouse modèle d'un ouvrier, et à la clé, l'éventualité d'une promotion sociale grâce à un mariage conclu avec un client aisé64 ?

En effet, s'il est vrai que les yanbua n'exercent pas une profession prestigieuse, elle peut pourtant permettre à des jeunes femmes, en particulier si elles officient dans des fumeries de haut de gamme, de devenir la concubine d'un des hommes riches qui y ont leurs habitudes, exactement comme des prostituées dans des bordels de luxe ${ }^{65}$. Les lecteurs sont probablement friands de ce genre d'histoires, ce qui explique le nombre assez important d'articles qui leur sont consacrés. L'histoire de la jeune He Zhen est représentative. He Zhen travaille comme prostituée à Chencun, un bourg situé au sud de Canton. Elle épouse un marchand, mais très vite les affaires de ce dernier périclitent en raison de son opiomanie. Le ménage se trouve réduit à une indigence extrême devant laquelle le mari montre une totale apathie. Elle parvient alors à le convaincre de la laisser s'engager comme yanbua dans une grande fumerie, où ses attraits et sa galanterie la rendent très vite célèbre. Pendant quelque temps, elle entretient son époux. Puis, une fois que son nouveau prestige le lui permet, elle devient la concubine d'un jeune homme fortuné qui rachète sa liberté à prix d'or au mari. Cette jeune femme, confrontée à une impasse dans son projet d'ascension sociale, utilise donc consciemment le métier de yanbua pour tirer parti de sa beauté et prendre place dans une famille riche ${ }^{66}$.

Les yanbua, du point de vue des facilités qu'offre leur métier aux entreprises de séduction intéressées, se situent à mi-chemin entre les prostituées et les serveuses de maisons de thé ou de restaurants. Ces dernières, même si elles sont réputées flirter avec les clients, opèrent

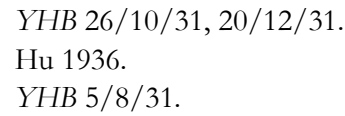


dans un espace dépourvu de toute possibilité d'intimité ${ }^{67}$. Elles ne bénéficient pas des occasions offertes par les longs tête-à-tête avec le client le temps de la préparation des pipes, qui peuvent avoir lieu dans un cabinet particulier s'il s'agit d'une fumerie de luxe. À l'opposé, les prostituées dont tout le métier s'organise autour de la séduction sont, plus que les yanbua, susceptibles de finir par convoler avec un de leurs clients.

Même si l'argumentaire mis en avant dans les sources consultées évoque surtout leur « indécence » et le travers consistant à détourner les hommes vers la drogue, on peut donc aussi discerner, pas vraiment explicitées, une autre série de craintes qui tournent, elles, autour de la transgression. Outre le fait qu'il offre l'opportunité à des jeunes femmes d'origine très modeste de se faufiler, grâce à un mariage, dans une famille fortunée, le métier de yanbua comme d'autres à la même époque, ouvre des possibilités d'indépendance financière à certaines femmes.

On aurait pu imaginer que la consommation d'opium par les femmes constitue un sujet privilégié de préoccupation pour les Cantonais des années 1930. Tel n'est pas le cas : à la différence de ce qui pouvait transparaître de certaines sources de la fin des Qing, les fumeuses d'opium, en nombre infime, ne font plus peur à personne. Les inquiétudes de la société à l'époque se portent sur les femmes qui instrumentalisent la drogue: les yanhua, ces jeunes employées qui préparent les pipes pour les clients des fumeries. Au-delà de leur assimilation à des prostituées se noue un faisceau de craintes liées en fait au maintien de l'ordre et, plus encore, de la hiérarchie établis. En dernière analyse, en effet, les séductions conjuguées de la drogue et de l'attrait physique de ces femmes apparaissent de nature à favoriser une mobilité sociale de mauvais aloi, qu'elle soit descendante (ruine du fumeur causée par les dépenses inconsidérées), ou ascendante (mariage de yanbua avec un riche client).

67 Chin 2007. 
Les yanhua, après leur brève éclipse du début des années 1930, demeurent partie intégrante de la vie des fumeries cantonaises jusqu'à l'éradication de l'opium par le Parti Communiste Chinois au début des années $1950^{68}$.

\section{Bibliographie}

Bourdieu Pierre, 1979, La distinction. Critique sociale du jugement, Paris, Les éditions de Minuit.

British Parlementary Papers, 1971, Dublin, Irish University Press.

CHIN Angelina, 2007, «Labor stratification and gendered subjectivities in the service industries of South China in the 1920s and 1930s: The case of nü zhaodai », Jindai funü shi yanjï (Recherches sur l'histoire des femmes à l'époque contemporaine), 14, p. $125-178$.

FIELD Andrew, 2000, «Dance Hall Culture and Urban Politics in Shanghai », thèse de doctorat, Columbia University.

GUANGZHOUSHI JINYAN WEIYUANHUI, 1937, Guangæhoushi jinyan weiyuanhui gongzuo jizyao (Résumé des activités du Comité d'interdiction de l'opium de la ville de Canton), Canton.

Henriot Christian, 1997, Belles de Shanghai, prostitution et sexualité à Shanghai aux XIX'XXe siècles (1849-1958), Paris, CNRS éditions.

Hu Puan, 1936, Zhonghua quanguo fengsu zhi (Recueil des us et coutumes de toute la Chine), Shanghai, Dada tushu gongyingshe.

LEE Edward Bing Shuey, 1936, Modern Canton, Shanghai, The Mercury Press.

LingNan Shehui Yanjiusuo, 1934, Shanan danmin diaocha baogao (Rapport d'enquête sur les Tankas de Shanan), Canton.

LuO Liming, 1994, Tangxi buayue hen (Nostalgie des merveilles de Tangxi), Hong Kong, Mingbao chubanshe.

MCMAHON Keith, 2002, The Fall of the God of Money: Opium Smoking in Nineteenth-Century China, Lanham, Rowman \& Littlefield.

Murayama Shigeru, 1941, Kanton Sadan (Quelques propos sur Canton), Osaka, Kibunkan.

PAULÈs Xavier, 2005a, «In search of smokers : a study of Canton opium smokers in the 1930s ", East Asian History, 29, p. 107-128.

—, 2005b, «L'opium à Canton 1912-1937. Essais de mainmise politique et pratiques sociales ", thèse de doctorat, Université Lyon 2.

68 Minzoku Taiwan (Le folklore taïwanais), vol. 3, nº (août 1943), p. 34-38; Entretien avec M. Mai Zhaoshen du 15/7/08. 
Pu Songling, 1996, Chroniques de l'étrange (traduction A. Levy), Arles, Philippe Picquier.

Royal COMmission ON OpIUM, 1894, Report of the Royal Commission on opium, Londres, Eyre and Spottiswoode, tome 5.

SIMA Qian, 1967, Mémoires bistoriques (traduction E. Chavannes), Paris, Adrien Maisonneuve.

Wang Di, 2003, Street Culture in Chengdu. Public Space, Urban Commoners, and Local Politics, 1870-1930, Stanford, Stanford University Press.

YANG Wanxiu, ZHONG Zhuoan, 1996, Guangz̧hou jianshi (Abrégé d'histoire de Canton), Canton, Guangdong renmin chubanshe.

ZHANG Ziping, 1994, «Gongzhai weiyuan » (Le commissaire de la dette publique), in Zhang Ziping xiaoshuo (Romans de Zhang Ziping), Guangzhou, Huacheng chubanshe.

ZHeng Yangwen, 2005, The Social Life of Opium in China, Cambridge, Cambridge University Press.

Zhu Qingbao, JiANG Qiuming, ZHANG Shijie, 1995, Yapian yu jindai zhongguo (L'opium et la Chine contemporaine), Nanjing, Jiangsu jiaoyu chubanshe. 